\title{
A Remark on Singular Perturbation Methods via the Lyapunov-Schmidt Reduction
}

By

\author{
Masaharu TANIGUCHI *
}

\begin{abstract}
For some reaction-diffusion equations, Lyapunov-Schmidt reduction was shown to be applicable to construct singularly perturbed equilibrium solutions. For this application, it is indispensable to show that some inverse operator are uniformly bounded. In this paper, we give an elementary proof of this fact.
\end{abstract}

\section{§ 1. Introduction}

For differential equations containing a small parameter in the spatial derivatives, there often exist solutions with internal transition layers. Hale and Sakamoto [1] applied Lyapunov-Schmidt reduction to construct singularly perturbed equilibrium solutions to Equation (1) below. This method also gave the stability condition for the solutions simultaneously.

In the following, we briefly sketch the method of [1], with special attention to the part where our present contribution appears. We consider the following equation

$$
\begin{gathered}
u_{t}=\varepsilon^{2} u_{x x}+f(u, x) \quad-1<x<1, t>0, \\
u_{x}(-1, t)=0=u_{x}(1, t) . \quad t>0 .
\end{gathered}
$$

The function $f$ satisfies the following assumptions.

1. $f: \boldsymbol{R} \times[-1,1] \rightarrow \boldsymbol{R}$ is of class $C^{\infty}$ with $f(0, x) \equiv 0, f(1, x) \equiv 0$.

2. There exists a positive constant $\beta$ such that

$$
f_{u}(0, x) \leq-3 \beta^{2}, \quad f_{u}(1, x) \leq-3 \beta^{2} \quad \text { for }-1 \leq x \leq 1
$$

Received July 15, 1994. Revised October 28, 1994.

1991 Mathematics Subject Classification: 35B25, 35K55.

Partially supported by the Sumitomo Foundation

* Research Institute for Mathematical Sciences, Kyoto University, Kyoto 606-01, Japan 
3. Let $J(x)=\int_{0}^{1} f(s, x) d s$. Then $J(0)=0$ with $J^{\prime}(0) \neq 0$, and $\int_{0}^{u} f(s, 0) d s<0$ for $u \in(0,1)$.

To construct unknown equilibrium solution $u$ of (1), we begin with a smooth approximate equilibrium solution $U(x, \varepsilon)$ of (1) that exhibits a transition layer at $x=0$. We denote $u-U(x, \varepsilon)$ by $u$. Then $u$ must satisfy

$$
0=\mathscr{L}^{\varepsilon} u+G(\varepsilon)+F(u, \varepsilon)
$$

where

$$
\begin{gathered}
\mathscr{L}^{\varepsilon} u=\varepsilon^{2} u_{x x}+f_{u}(U(x, \varepsilon), x) u, \\
G(\varepsilon)(x)=\varepsilon^{2} U_{x x}(x, \varepsilon)+f(U(x, \varepsilon), x) \\
F(u, \varepsilon)=f(U(x, \varepsilon)+u, x)-f(U(x, \varepsilon), x)-f_{u}(U(x, \varepsilon), x) u .
\end{gathered}
$$

The approximate solution $U(x, \varepsilon)$ is constructed to satisfy $\sup _{x \in[-1,1]}|G(\varepsilon)(x)|$ $=O\left(\varepsilon^{2}\right)$. The operator $\mathscr{L}^{\varepsilon}$ turns out to have exactly one eigenvalue $\lambda_{1}(\varepsilon)$ that approaches zero as $\varepsilon \rightarrow 0$, and there are some $\varepsilon_{0}>0, \mu_{0}>0$ such that the remaining eigenvalues are less than $-\mu_{0}$ for $0<\varepsilon \leq \varepsilon_{0}$. Moreover the eigenspace $\lambda_{1}(\varepsilon)$ is one-dimensional, and $\lambda_{1}(\varepsilon)=k_{1} \varepsilon+o(\varepsilon)$ as $\varepsilon \rightarrow 0$ for some constant $k_{1}$. Let $Y=$ $C[-1,1]$ with the norm

$$
|h|_{0}=\sup _{\underline{x} \in[-1,1]}|h(x)| \quad \text { for } h \in Y,
$$

and let $X=\left\{u \in C^{2}[-1,1] ; u_{x}(-1)=0=u_{x}(1)\right\}$ with the norm

$$
|u|_{2, \varepsilon}=|u|_{0}+\varepsilon\left|u_{x}\right|_{0}+\varepsilon^{2}\left|u_{x x}\right|_{0} \quad \text { for } u \in X
$$

Let $\phi_{1}(\varepsilon)$ be an eigenfunction associated with $\lambda_{1}(\varepsilon)$ normalized as (12). Let $E$ be the following projection onto the span of $\phi_{1}(\varepsilon)$ :

$$
E u=\int_{-1}^{1} u(x) \phi_{1}(x, \varepsilon) d x \phi_{1}(\cdot, \varepsilon) /\left\|\phi_{1}(\cdot, \varepsilon)\right\|_{L^{2}(-1,1)}^{2},
$$

and let $Y_{1}, X_{1}$ be the null spaces of $E$ in $Y, X$, respectively. Using the decompositions

$$
Y=\operatorname{span}\left\{\phi_{1}(\varepsilon)\right\} \oplus Y_{1}, \quad X=\operatorname{span}\left\{\phi_{1}(\varepsilon)\right\} \oplus X_{1}
$$

we split (2) into

$$
\lambda_{1}(\varepsilon) \alpha \phi_{1}(\varepsilon)+E G(\varepsilon)+E F\left(\alpha \phi_{1}(\varepsilon)+w, \varepsilon\right)=0,
$$




$$
\mathscr{L}^{\varepsilon} w+(I-E) G(\varepsilon)+(I-E) F\left(\alpha \phi_{1}(\varepsilon)+w, \varepsilon\right)=0,
$$

where $u=\alpha \phi_{1}(x, \varepsilon)+w$ with $\alpha \in \boldsymbol{R}, w \in X_{1}$. Hale and Sakamoto [1] solved (7) as $w=w^{*}(\alpha, \varepsilon)$ with

$$
\left|w^{*}(\alpha, \varepsilon)\right|_{2, \varepsilon}=O\left(\alpha^{2}+\varepsilon^{2}\right) \quad \text { as }|\alpha|+\varepsilon \rightarrow 0
$$

by estimating the second and the third terms of (7) and using the contraction mapping principle. We substitute $w=w^{*}(\alpha, \varepsilon)$ into (6), and obtain an equation for $\alpha$, which we denote by $B(\alpha, \varepsilon)=0$. Using the fact $|G(\varepsilon)|_{0}=O\left(\varepsilon^{2}\right)$ and $\lambda_{1}(\varepsilon)=k_{1} \varepsilon+o(\varepsilon)$, we can solve $B(\alpha, \varepsilon)=0$ as $\alpha=\alpha^{*}(\varepsilon)$. Here $\alpha^{*}(\varepsilon)=O(\varepsilon)$ as $\varepsilon \downarrow 0$. Now the desired equilibrium solution of (1) is obtained by

$$
u=U(x, \varepsilon)+\alpha^{*}(\varepsilon) \phi_{1}(x, \varepsilon)+w^{*}\left(\alpha^{*}(\varepsilon), \varepsilon\right) .
$$

In order to obtain the crucial estimate (8), Hale and Sakamoto [1] used the fact that $\left(\mathscr{L}^{\varepsilon}\right)^{-1}: Y_{1} \rightarrow Y_{1}$ is bounded uniformly in $\varepsilon \in\left(0, \varepsilon_{0}\right]$. If the topology of $Y_{1}$ is that of $L^{2}(-1,1)$, it suffices for the boundedness that there are no eigenvalues of $\left.\mathscr{L}^{\varepsilon}\right|_{Y_{1}}$ around 0 , which is known for many years. However, now that $Y_{1}$ is a subspace of $C[-1,1]$, it is not obvious for the author to conclude the uniform boundedness directly from the distribution of eigenvalues.

The main issue of this paper is to give a proof to the fact stated above.

Theorem 1. The operator $\left(\mathscr{L}^{\varepsilon}\right)^{-1}: Y_{1} \rightarrow Y_{1}$ is bounded uniformly in sufficiently small $\varepsilon>0$.

\section{§ 2. Preliminaries}

We describe how to construct $U(x, \varepsilon)$ and collect properties of $\mathscr{L}^{\varepsilon}$ following Hale and Sakamoto [1].

Let $\zeta_{0}(x), \zeta_{+}(x)$ be cutoff functions of class $C^{\infty}[-1,1]$ with

$$
\zeta_{0}(x)=\left\{\begin{array}{ll}
1 & |x| \leq 1 / 4 \\
0 & |x| \geq 1 / 2 \\
0 \leq \zeta_{0}(x) \leq 1 & x \in[-1,1],
\end{array} \quad \zeta_{+}(x)= \begin{cases}0 & x \in[-1,0] \\
1-\zeta_{0}(x) & x \in[0,1] .\end{cases}\right.
$$

$U(x, \varepsilon)$ is given by

$$
U(x, \varepsilon)=\zeta_{0}(x)\left\{z_{0}(\eta)+\varepsilon z_{1}(\eta)\right\}+\zeta_{+}(x),
$$

where $\eta=x / \varepsilon$. Here $z_{0}, z_{1}$ are some smooth and bounded functions on $\boldsymbol{R}$. In particular $z_{0}$ satisfies $d^{2} z_{0} / d \eta^{2}+f\left(z_{0}(\eta), 0\right)=0$ with $z_{0}(-\infty)=0, z_{0}(\infty)=1$ and 


$$
\left|\frac{d z_{0}}{d \eta}(\eta)\right| \leq k_{0} \exp (-2 \beta|\eta|)
$$

with some constant $k_{0}>0$. Then $U(x, \varepsilon)$ satisfies $|G(\varepsilon)|_{0}=O\left(\varepsilon^{2}\right)$ as in Lemma 2.1 of $[1]$.

Let $\lambda_{1}(\varepsilon)$ be the principal eigenvalue of $\mathscr{L}^{\varepsilon}$, and let $\phi_{1}(\varepsilon)$ be the eigenfunction associated with $\lambda_{1}(\varepsilon)$ normalized as

$$
\phi_{1}(0, \varepsilon)=d z_{0} / d \eta(0)
$$

Proposition 1 (Theorem 3.1 and Lemma 3.4 in [1]). There exists $\varepsilon_{0}>0$ such that the following assertions are valid:

(i) $\lambda_{1}(\varepsilon)$ is simple for $0<\varepsilon<\varepsilon_{0}$, and satisfies $\lim _{\varepsilon \rightarrow 0} \lambda_{1}(\varepsilon) / \varepsilon=k_{1}$, where

$$
k_{1}=-J^{\prime}(0) /\left\|d z_{0} / d \eta\right\|_{L^{2}(R)}^{2} .
$$

(ii) There exists a constant $\mu_{0}>0$ such that, all other eigenvalues of $\mathscr{L}^{\varepsilon}$ are less than $-\mu_{0}$.

(iii) There exists a constant $k_{2}>0$ such that $\left|\phi_{1}(x, \varepsilon)\right| \leq k_{2} \exp (-2 \beta|x| / \varepsilon)$ for $0<\varepsilon<\varepsilon_{0}$.

(iv) Let $\tilde{\phi}_{1}(\eta, \varepsilon)=\phi_{1}(\varepsilon \eta, \varepsilon)$. Then $\tilde{\phi}(\cdot, \varepsilon)$ converges to $d z_{0} / d \eta$ in $C^{2}(K)$ as $\varepsilon \rightarrow 0$, where $K$ is any compact set in $\boldsymbol{R}$.

Remark 1. The equilibrium solution (1) is stable if $k_{1}<0$, and is unstable if $k_{1}>0$. See [1] for the proof.

There exists a constant $k_{3}>0$ such that $|E u|_{0} \leq k_{3}|u|_{0}$ for $u \in Y$, $0<\varepsilon<\varepsilon_{0}$.

\section{§ 3. Proof of Theorem 1}

The following arguments are similar to those given by $\mathrm{Ni}$ and Takagi [2], where the inverse operator of another elliptic operator is studied.

We prove by contradiction. Assume that there exist $\varepsilon_{1}>\varepsilon_{2}>\cdots$ $>\varepsilon_{j}>\cdots>0$ with $\lim _{j \rightarrow \infty} \varepsilon_{j}=0$ such that there exist $\phi_{j} \in X_{1}$ and $h_{j} \in Y_{1}$ satisfying

$$
\begin{aligned}
& \mathscr{L}^{\varepsilon} \phi_{j}=h_{j} \quad \text { in }(-1,1), \\
& \frac{d \phi_{j}}{d x}(-1)=0=\frac{d \phi_{j}}{d x}(1),
\end{aligned}
$$

with 


$$
\left|\phi_{j}\right|_{0}=1, \quad\left|h_{j}\right|_{0}<1 / j
$$

for each $j=1,2, \cdots$. Let

$$
\begin{aligned}
& \tilde{\phi}_{j}(\eta)=\phi_{j}\left(\varepsilon_{j} \eta, \varepsilon_{j}\right) \\
& \tilde{h}_{j}(\eta)=h_{j}\left(\varepsilon_{j} \eta, \varepsilon_{j}\right) \\
& \tilde{U}_{j}\left(\eta, \varepsilon_{j}\right)=U_{j}\left(\varepsilon_{j} \eta, \varepsilon_{j}\right)
\end{aligned} \quad \text { for } \eta \in\left(-1 / \varepsilon_{j}, 1 / \varepsilon_{j}\right) .
$$

Then

$$
\frac{d^{2} \tilde{\phi}_{j}}{d \eta^{2}}+f_{u}\left(\tilde{U}\left(\eta, \varepsilon_{j}\right), \varepsilon_{j} \eta\right) \tilde{\phi}_{j}=\tilde{h}_{j}
$$

in $\left(-1 / \varepsilon_{j}, 1 / \varepsilon_{j}\right)$. Let $K=[-n, n]$ for arbitrarily fixed $n \in N$. We consider (15) in $K$ for sufficiently large $j$. Since $\left|\phi_{j}\right|_{0}=1$, the sequence $\left\{\tilde{\phi}_{j}\right\}$ remains bounded in $C(K)$. We can also see that

$$
f_{u}\left(\tilde{U}\left(\eta, \varepsilon_{j}\right), \varepsilon_{j} \eta\right)=f_{u}\left(\zeta_{0}\left(\varepsilon_{j} \eta\right)\left\{z_{0}(\eta)+\varepsilon_{j} z_{1}(\eta)\right\}+\zeta_{+}\left(\varepsilon_{j} \eta\right), \varepsilon_{j} \eta\right)
$$

is bounded in $C(K)$ uniformly in $j$. The sequence $\left\{\tilde{h}_{j}\right\}$ is bounded in $C(K)$. Hence from (15), $\left\{d^{2} \tilde{\psi} / d \eta^{2}\right\}$ is also bounded in $C(K)$. From the following interpolation inequality

$$
\left\|\frac{d \tilde{\phi}_{j}}{d \eta}\right\|_{C(K)} \leq \delta\left\|\frac{d^{2} \tilde{\phi}_{j}}{d^{2} \eta}\right\|_{C(K)}+\frac{2}{\delta}\left\|\tilde{\phi}_{j}\right\|_{C(K)}
$$

for every $\delta>0$, the sequence $\left\{\tilde{\phi}_{j}\right\}_{j=1}^{\infty}$ remains bounded in $C^{2}(K)$. For any $\theta \in(0,1)$, the imbedding $C^{2}(K) \subset C^{1, \theta}(K)$ is compact. By taking a subsequence, we can assume that $\left\{\tilde{\phi}_{j}\right\}_{j=1}^{\infty}$ converges in $C^{1, \theta}(K)$. As $j \rightarrow \infty$, we have

$$
\begin{aligned}
f_{u}\left(\tilde{U}\left(\eta, \varepsilon_{j}\right), \varepsilon_{j} \eta\right) & \rightarrow f_{u}\left(z_{0}(\eta), 0\right), \\
\tilde{h}_{j}(\eta) & \rightarrow 0
\end{aligned}
$$

uniformly in $\eta \in K$. Thus $d^{2} \tilde{\phi}_{j} / d \eta^{2}(\eta)$ converges uniformly in $\eta \in K$. It follows that $\left\{\tilde{\phi}_{j}\right\}$ converges in $C^{2}(K)$. Let $\tilde{\phi}_{\infty}=\lim _{j \rightarrow \infty} \tilde{\phi}_{j}$ in $C^{2}(K)$. Then $\left\|\tilde{\phi}_{\infty}\right\|_{C(K)} \leq 1$.

We apply the argument stated above for each $K=[-n, n]$ with $n=1,2, \cdots$. Then it turns out that $\tilde{\phi}_{\infty}$ is a bounded function of class $C^{\infty}(\boldsymbol{R})$ and satisfies

$$
\frac{d^{2} \tilde{\phi}_{\infty}}{d \eta^{2}}+f_{u}\left(z_{0}(\eta), 0\right) \tilde{\phi}_{\infty}(\eta)=0 \quad \text { in } \quad \boldsymbol{R}
$$

This equation has two linearly independent solutions, that is, 


$$
\frac{d z_{0}}{d \eta}(\eta), \quad \frac{d z_{0}}{d \eta}(\eta) \int_{0}^{\eta}\left(\frac{d z_{0}}{d \eta}(\eta)\right)^{-2} d \eta
$$

The latter one goes to infinity as $\eta \rightarrow \infty$. Since $\tilde{\phi}_{\infty}$ is a bounded solution, there must be a constant $c$ such that

$$
\tilde{\phi}_{\infty}(\eta)=c \frac{d z_{0}}{d \eta}(\eta)
$$

Here $c \neq 0$ follows from the next lemma, the proof of which is stated at the end of $\S 3$.

Lemma 1. Let an arbitrary $x$ with $\left|\phi_{j}(x)\right|=1$ be denoted by $x_{j}$. Then $x_{j} / \varepsilon_{j}$ must remain bounded as $j \rightarrow \infty$.

Indeed, taking a subsequence, $x_{j} / \varepsilon_{j}$ converges to some $\eta_{\infty} \in \boldsymbol{R}$. From

$$
\left|\tilde{\phi}_{j}\left(x_{j} / \varepsilon_{j}\right)\right|=\left|\phi_{j}\left(x_{j}\right)\right|=1,
$$

we have $\left|\tilde{\phi}_{\infty}\left(\eta_{\infty}\right)\right|=1$. Hence $c \neq 0$.

Since $\phi_{j} \in X_{1}$, we have $\int_{-1}^{1} \phi_{j}(x) \phi_{1}(x, \varepsilon) d x=0$, that is,

$$
\int_{-\frac{1}{\varepsilon_{j}}}^{\frac{1}{\varepsilon_{j}}} \tilde{\phi}_{j}(\eta) \tilde{\phi}_{1}\left(\eta, \varepsilon_{j}\right) d \eta=0
$$

Combining $\left|\tilde{\phi}_{1}\left(\eta, \varepsilon_{j}\right)\right| \leq k_{2} \exp (-2 \beta|\eta|)$ and $\left\|\tilde{\phi}_{j}\right\|_{C\left[-1 / \varepsilon_{j}, 1 / \varepsilon_{j}\right]}=1$, we have

$$
\left.\mid \tilde{\phi}_{j}(\eta)\right) \tilde{\phi}_{1}\left(\eta, \varepsilon_{j}\right) \mid \leq k_{2} \exp (-2 \beta|\eta|) \text {. }
$$

From Lebesgue's dominated convergence theorem,

$$
\int_{-\infty}^{\infty} \tilde{\phi}_{\infty}(\eta) z_{0}(\eta) d \eta=0
$$

which contradicts (16) when $c \neq 0$. This completes the proof of Theorem 1 .

Proof of Lemma 1. We prove by contradiction. Assume the contrary. Then by taking a subsequence, we have

$$
\lim _{j \rightarrow \infty} x_{j} / \varepsilon_{j}=\infty \text { or } \lim _{j \rightarrow \infty} x_{j} / \varepsilon_{j}=-\infty .
$$

Without loss of generality, we assume $\lim _{j \rightarrow \infty} x_{j} / \varepsilon_{j}=\infty$. Let 


$$
\begin{aligned}
\hat{\phi}_{j}(\eta) & =\phi_{j}\left(\varepsilon_{j} \eta+x_{j}\right) \\
\hat{h}_{j}(\eta) & =h_{j}\left(\varepsilon_{j} \eta+x_{j}\right) \quad \text { for }\left(-1-x_{j}\right) / \varepsilon_{j} \leq \eta \leq\left(1-x_{j}\right) / \varepsilon_{j} . \\
\hat{U}\left(\eta, \varepsilon_{j}\right) & =U_{j}\left(\varepsilon_{j} \eta+x_{j}, \varepsilon_{j}\right) .
\end{aligned}
$$

These functions can be defined at least for $-1 \leq \eta \leq 0$ for sufficiently large $j$. From (14),

$$
\frac{d^{2} \hat{\psi}_{j}}{d \eta^{2}}+f_{u}\left(\hat{U}\left(\eta, \varepsilon_{j}\right), \varepsilon_{j} \eta+x_{j}\right) \hat{\phi}_{j}=\hat{h}_{j} \quad \text { in }(-1,0)
$$

Using $\left|\phi_{j}\left(x_{j}\right)\right|=1$ and $d \psi_{j} / d x\left(x_{j}\right)=0$, we have

$$
\frac{d \hat{\psi}_{j}}{d \eta}(0)=0, \quad\left|\hat{\psi}_{j}(0)\right|=1 \text {. }
$$

By taking a subsequence, we can assume $\left\{x_{j}\right\}_{j=1}^{\infty}$ converges to some $x_{\infty} \in[-1,1]$. From $\lim _{j \rightarrow \infty} x_{\jmath} / \varepsilon_{\jmath}=\infty$, we have $\varepsilon_{\jmath} \eta+x_{j}>0$. Hence

$$
\zeta_{0}\left(\varepsilon_{j} \eta+x_{j}\right)+\zeta_{+}\left(\varepsilon_{j} \eta+x_{j}\right)=1
$$

Using $\lim _{j \rightarrow \infty} x_{j} / \varepsilon_{j}=\infty$ again, we obtain

$$
z_{0}\left(\eta+x_{j} / \varepsilon_{j}\right)+\varepsilon_{j} z_{1}\left(\eta+x_{j} / \varepsilon_{j}\right) \rightarrow 1,
$$

uniformly in $\eta \in[-1,0]$. Therefore

$$
\widehat{U}\left(\eta, \varepsilon_{\jmath}\right)=\zeta_{0}\left(\varepsilon_{j} \eta+x_{\jmath}\right)\left\{z_{0}\left(\eta+x_{j} / \varepsilon_{j}\right)+\varepsilon_{j} z_{1}\left(\eta+x_{j} / \varepsilon_{j}\right)\right\}+\zeta_{+}\left(\varepsilon_{j} \eta+x_{j}\right)
$$

converges to 1 uniformly in $-1 \leq \eta \leq 0$. By quite the same argument as in the proof of Theorem 1 , we can assume $\hat{\phi}_{j}$ converges to some $\hat{\phi}_{\infty}$ in $C^{2}[-1,0]$. The function $\hat{\psi}_{\infty}$ satisfies

$$
\begin{gathered}
\frac{d^{2} \hat{\psi}_{\infty}}{d \eta^{2}}+f_{u}\left(1, x_{\infty}\right) \hat{\psi}_{\infty}=0 \quad \text { in }(-1,0), \\
\frac{d \hat{\psi}_{\infty}}{d \eta}(0)=0, \quad\left|\hat{\psi}_{\infty}(0)\right|=1, \\
\left|\hat{\phi}_{\infty}(\eta)\right| \leq 1 \quad \text { in }[-1,0] .
\end{gathered}
$$

By the assumptions for $f, f_{u}\left(1, x_{\infty}\right)<0$. This contradicts the maximum principle (see for example, [3]). The proof of Lemma 1 is completed. 


\section{§ 4. Uniform Boundedness of Another Inverse Operator}

In Sakamoto [4], the following system of equations

$$
\begin{aligned}
& u_{t}=\varepsilon^{2} u_{x x}+f(u, v) \\
& v_{t}=\frac{1}{\sigma} v_{x x}+g(u, v)
\end{aligned} \quad \text { in } 0<x<1, t>0,
$$

with the Neumann boundary condition

$$
\begin{aligned}
& u_{x}(0, t)=0=u_{x}(1, t) \\
& v_{x}(0, t)=0=v_{x}(1, t)
\end{aligned} \quad t>0
$$

is studied. For the assumption of $f$ and $g$, see $\S 1$ of [4]. Singularly perturbed equilibrium solutions of (17) are constructed in [4] for every $(\varepsilon, \sigma) \in$ $\left(0, \varepsilon_{0}\right] \times\left(0, \sigma_{0}\right]$ via the Lyapunov-Schmidt reduction. Here $\varepsilon_{0}, \sigma_{0}$ are some positive constants. Let

$$
L^{\varepsilon, \sigma}=\varepsilon^{2} \frac{d^{2}}{d x^{2}}+f_{u}(U(x, \varepsilon, \sigma), V(x, \varepsilon, \sigma))
$$

where $U(x, \varepsilon, \sigma), V(x, \varepsilon, \sigma)$ are approximate equilibrium solutions of (17) as in $\S 2$ of [4]. We denote a complete orthonormal system in $L^{2}(0,1)$ of eigenfunctions and eigenvalues by $\left\{\phi_{n}(\varepsilon, \sigma), \lambda_{n}(\varepsilon, \sigma)\right\}$, where $\lambda_{1}(\varepsilon, \sigma)>\lambda_{2}(\varepsilon, \sigma) \geq$ $\lambda_{3}(\varepsilon, \sigma) \geq \cdots$. The principal eigenvalue $\lambda_{1}(\varepsilon, \sigma)$ approaches zero as $\varepsilon \rightarrow 0$, and there exists a constant $\mu_{1}>0$ such that all other ones are less than $-\mu_{1}$. Let $E$ denote the orthogonal projection onto span $\left\{\phi_{1}(\varepsilon, \sigma)\right\}$, that is, $E u=$ $\left(u, \phi_{1}(\varepsilon, \sigma)\right)_{L^{2}(0,1)} \phi_{1}(\varepsilon, \sigma)$.

The following assertion is a part of Corollary 3.2 of [4].

Theorem 2. The linear operator

$$
\left(L^{\varepsilon, \sigma}\right)^{-1}(I-E): C[0,1] \rightarrow C[0,1]
$$

is bounded uniformly in $(\varepsilon, \sigma) \in\left(0, \varepsilon_{0}\right] \times\left(0, \sigma_{0}\right]$. Here I is the identical operator.

The argument as in the proof of Theorem 1 is valid to prove Theorem 2 .

Proof of Theorem 2. We prove by contradiction. Assume there exist $\varepsilon_{1}>\cdots>\varepsilon_{j}>\cdots>0$ with $\lim _{j \rightarrow \infty} \varepsilon_{j}=0$ and $\left\{\sigma_{j}\right\}_{j=1}^{\infty} \subset\left(0, \sigma_{0}\right]$ such that the following holds. There exist $\phi_{j}$ and $h_{j}$ satisfying

$$
\left(\varepsilon_{j}\right)^{2} \frac{d^{2} \phi_{j}}{d x^{2}}+f_{u}\left(U\left(x, \varepsilon_{j}, \sigma_{j}\right), V\left(x, \varepsilon_{j}, \sigma_{j}\right)\right) \phi_{j}=h_{j} \quad \text { in }(0,1),
$$




$$
\frac{d \psi_{j}}{d x}(0)=0=\frac{d \psi_{j}}{d x}(1)
$$

with $\left|\phi_{j}\right|_{0}=1,\left|h_{j}\right|_{0}<1 / j$, and $\int_{0}^{1} h_{j}(x) \phi_{1}\left(\varepsilon_{j}, \sigma_{j}\right) d x=0$. Since $L^{\varepsilon, \sigma}$ is self-adjoint, we have

$$
\int_{0}^{1} \phi_{j}(x) \phi_{1}\left(\varepsilon_{j}, \sigma_{\jmath}\right) d x=0
$$

If we replace Lemma 1 by the following lemma and Proposition 1 by Lemma 3.1 of [4] respectively, the similar argument for Theorem 1 is applicable. We omit the detail.

Let $x^{*}\left(\sigma_{j}\right)$ is as in $\S 2$ of Sakamoto [4]. Corresponding Lemma 1, we have the following:

Lemma 2. Let an arbitrary $x$ with $\left|\phi_{j}(x)\right|=1$ be denoted by $x_{j}$. Then $\left(x_{j}-x^{*}\left(\sigma_{j}\right)\right) / \varepsilon$, remains bounded as $j \rightarrow \infty$.

Proof of Lemma 2. We only sketch the proof. Assume the contrary. Without loss of generality, we can assume $\lim _{j \rightarrow \infty}\left(x_{j}-x^{*}\left(\sigma_{j}\right)\right) / \varepsilon_{j}=\infty$. Taking a subsequence, we can also assume $\left\{x_{\jmath}\right\}_{j=1}^{\infty}$ converges to some $x_{\infty} \in[0,1]$, and that $\left\{\sigma_{j}\right\}_{j=1}^{\infty}$ converges to $\sigma_{\infty} \in\left[0, \sigma_{0}\right]$. Let $\hat{\psi}_{j}(\eta)=\phi_{j}\left(\varepsilon, \eta+x_{j}\right)$, which can be defined at least on $[-1,0]$ for sufficiently large $j$. By the same argument as in the proof of Lemma 1 , we can take a subsequence such that $\left\{\hat{\phi}_{j}\right\}$ converges to some $\hat{\phi}_{\infty}$ in $C^{2}[-1,0]$. The function $\hat{\psi}_{\infty}$ satisfies

$$
\begin{gathered}
\frac{d^{2} \hat{\psi}_{\infty}}{d \eta^{2}}+f_{u}\left(h_{+}\left(\hat{V}\left(x_{\infty}, \sigma_{\infty}\right)\right), \hat{V}\left(x_{\infty}, \sigma_{\infty}\right)\right) \hat{\psi}_{\infty}=0 \quad \text { in }(-1,0), \\
\frac{d \hat{\psi}_{\infty}}{d \eta}(0)=0, \quad\left|\hat{\psi}_{\infty}(0)\right|=1, \\
\left|\hat{\phi}_{\infty}(\eta)\right| \leq 1 \quad \text { in }[-1,0] .
\end{gathered}
$$

For the definitions for $h_{+}(\cdot)$ and $\widehat{V}$, see [4]. In view of the assumptions on $f$ in $\S 1$ of [4], we have

$$
\left.f_{u}\left(h_{+}\left(\hat{V}\left(x_{\infty}, \sigma_{\infty}\right)\right), \hat{V}\left(x_{\infty}, \sigma_{\infty}\right)\right)\right)<0
$$

which combined with (19) contradicts the maximum principle. The proof of Lemma 2 is completed. 


\section{Acknowledgement}

The author would like to thank the referee for giving many useful instructions.

\section{References}

[1] Hale, J. K. and Sakamoto, K., Existence and stability of transition layers, Japan J. Appl. Math., 5 (1988), 367-405.

[2] Ni, W.-M. and Takagi, I., Point-condensation generated by a reaction-diffusion system in axially symmetric domains, Japan J. Indus. Appl. Math., 12 (1995), 327-365.

[3] Protter, M. H. and Weinberger, H. F., Maximal principles in differential equations, Springer-Verlag, New York, Berlin, 1967.

[4] Sakamoto, K., Construction and stability analysis of transition layer solutions in reaction-diffusion systems, Tôhoku Math. J., 42 (1990), 17-44. 